\title{
79. Antidromically Evoked Cord Surface Potentials and Natural Sleep in the Cat
}

\author{
By Kisou Kubota, Takahide Kurokawa, Yoshiaki Mayanagi, \\ and Reisaku TANAKa \\ Department of Neurophysiology, Institute of Brain Research, \\ Faculty of Medicine, University of Tokyo \\ (Comm. by Yas Kuno, M.J.A., April 12, 1967)
}

The relation of the tonic muscular activities during the parasleep (paradoxical phase of sleep) ${ }^{1,4), 13}$ in the cat is associated with excitability decrease of the motoneuron. This was shown from threshold measurement of the motoneuron activation by a current applied directly through intraspinally implanted electrodes. ${ }^{5}$ However, potential recording of the motoneuron with use of the intraspinal electrodes did not reveal the corresponding change, i.e. evidence of the excitability decrease (cf. 13).

It was attempted to observe antidromically-activated surface potentials at the lumbar and sacral cords with special reference to the natural sleep. As previously reported ${ }^{8,9)}$ potentials originating from the motor axons, motoneuronal somas, dendrites and interneurons called as Renshaw cells are recordable from the cord surface. Lloyd termed these potentials as $m-, b-, d-$, and $l$-responses, respectively. ${ }^{8)}$ If recorded at the lateral surface of the cord, the potentials consist of the initial positive b-response, succeeding negative d-response, and later repetitive positive wavelets of Renshaw cells (Renshaw ripples). ${ }^{8)}$ Primary concern in this brief communication is whether these antidromic cord potentials are depressed or not during para-sleep. The para-sleep was differentiated from the usual orthosleep (sleep in slow wave and spindle burst in EEG), if the low voltage EEG, neck muscle silence, and rapid eye movements were observed. ${ }^{1,4)}$

During preliminary operations under the pentobarbital anesthesia (Nembutal; $35 \mathrm{mg} / \mathrm{kg}$ ) were implanted the screw electrodes for the cortical EEG, steel electrodes for the cervical EMG and collar type nerve electrodes for the peripheral nerves, such as the sciatic, peroneal, tibial. Laminectomy was done so that in the chronic state the dorsal and lateral sides of the lower lumbar and upper sacral cords may be visualized. Ventral roots of one side and dorsal roots of both sides were cut from L6 to S2. Extremely careful manipulations were necessary to obtain the satisfactory d-response. Excised dura 
and skin were closed. A specially designed metal frame, as described in the fusimotor study, ${ }^{6,7)}$ was permanently attached to the cat's back. This made the potential recording in the unanesthetized condition possible.

Observations were started in the following day after the animal recovered from the anesthesia. As described, ${ }^{6), 7)}$ a liquid paraffin pool was made after exposing the cord. Painful stimuli were avoided, using local anesthetics. To avoid the leakage of the CSF, small pieces of gelfoam were used in the space between the cord and edges of the excised duras. As with Lloyd, ${ }^{8)}$ the d-response was the largest at the lateral surface, particularly just dorsal of the denticulate ligament line.

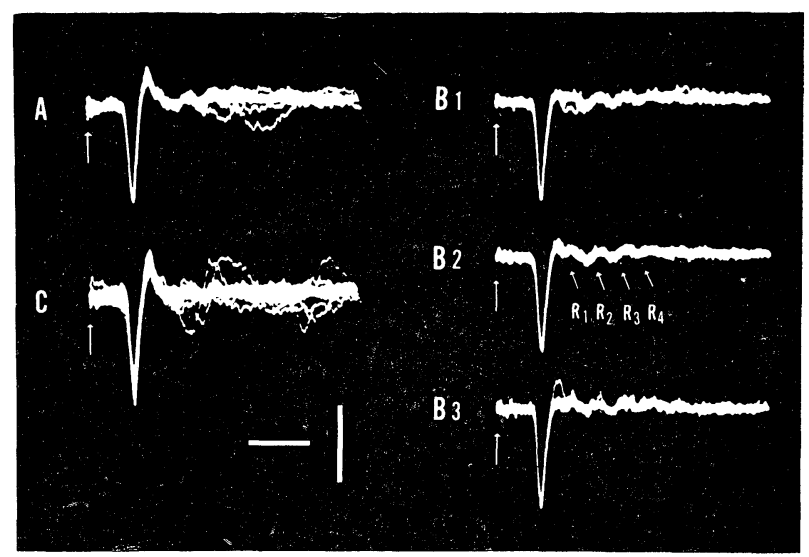

Fig. 1. Antidromically evoked surface potentials and natural sleep in the cat. The responses were recorded by a silver ball electrode placed upon a surface point, slightly dorsal to the denticulate ligament at the $\mathrm{L} 7 \mathrm{mid}$-segmental level. The reference was another silver ball electrode placed on the dorsal root entry zone of the other side at the same level. Upward deflection represents negativity at the active electrode. A: control ortho-sleep state. B1, 2, and 3: para-sleep. C: awake state immediately after the para-sleep. The sciatic nerve at the popliteal fossa was stimulated at an intensity of 3.7 multiples of the threshold of alpha fibers $(1 \mathrm{cps}, 0.01$ msec duration). All traces are superposed for 10 times. R1-4 of B2 indicate the successive positive peaks of the Renshaw ripples. Arrows at left indicate the onset time of the stimulus. Time, 2 msec and amplitude, $200 \mathrm{uV}$.

The size of the d-response recorded during ortho-sleep (Fig. 1A) and at the relaxed awake state (Fig. 1C) were quite stable, as far as tested at a low rate of the shock repetition. As soon as the animal fell into the para-sleep state, the d-response became smaller in size or was abolished almost completely without any changes in the size of positive b-responses. Fig. 1 B1 and B2 illustrate samples of 
the decreased responses during para-sleep. It occastonally reappeared (Fig. 1 B3). When the animal woke from the para-sleep, the $d-$ response reappeared instantaneously, being the size as large as that in the ortho-sleep. When the animal shows the behavior of motion, such as gazing, shoulder shaking and so on, the d-response could be larger for a while than that during ortho-sleep or relaxed awake state. This occurred, even when the supramaximal b-response was evoked.

As for Renshaw cell activity, there were no clear depressive changes in the ripple size, peak intervals, nor peak latencies during para-sleep. This negative result is shown in Fig. $1 \mathrm{~B} 2$.

The d-response is regarded as constituting of the conducting spike potential along the motoneuronal dendrite. And its conduction velocity was estimated as $5 \mathrm{~m} / \mathrm{sec}^{8}{ }^{8}$ Recent intracellular studies of the cat motoneurons revealed that its dendrite is capable of conducting the spike antidromically, but the intracellular counterpart of the surface d-response is not yet established. The delayed depolarization $^{3), 11}$ and/or the post-spike depolarization, ${ }^{10)}$ which are generated after the spike corresponding in time with the d-response generation, seems to be of different origin because of following two reasons. Firstly, the post-spike depolarization is observable even in complete absence of the d-response, occurring, for example, after the ventral axonotomy. ${ }^{10)}$ Secondly, the delayed depolarization and post-spike depolarization are seen intracellularly even under the pentobarbital anesthesia which depresses the d-response sensitively. ${ }^{10,11)}$ The depression of $d$-response during para-sleep indicates that the motoneulrona dendrites are not invaded antidromically, that is, the spike is not conducted. This is probably due to the membrane potential change either by the IPSP or by the EPSP, impinging during parasleep upon the motoneuron.

Summary. The d-response recorded from the cord surface after the antidromic shock was depressed during para-sleep, compared with the preceeding or succeeding ortho-sleep and/or relaxed awake state.

\section{References}

1) Dement, W.: The occurrence of low voltage, fast, encephalogram patterns during behavioral sleep in the cat. Electroenceph. Clin. Neurophysiol., 10, 291-296 (1958).

2) Eccles, J. C., Fatt, P., and Koketsu, K.: Cholinergic and inhibitory synapses in a pathway from motor-axon collaterals to motoneurons. J. Physiol., 126, 524-562 (1954).

3) Granit, R., Kernell, D., and Smith, R.S.: Delayed depolarization of mammalian 
motoneurones. J. Physiol., 168, 890-910 (1963).

4) Jouvet, M.: Recherches sur les structures nerveuses et les mecanismes responsables des differentes phases du sommeil physiologique. Arch. ital. Biol., 100, 125-206 (1962).

5) Kubota, K., and Kidokoro, Y.: Excitability of the membrane of lumbar motor neurons and natural sleep in the cat. Jap. J. Physiol., 16, 217-226 (1966).

6) Kubota, K., and Tanaka, R.: The fusimotor activity and natural sleep in the cat. Brain Research., 3, 198-201 (1966).

7) Kubota, K., Tanaka, R., and Tsuzuki, N.: Muscle spindle activity and natural sleep in the cat. Jap. J. Physiol. (1967) (in press).

8) Lloyd, D.P.C.: Electrical signs of impulse conduction in spinal motoneurons. J. gen. Physiol., 35, 255-288 (1951).

9) - - After-currents, after-potentials, excitability, and ventral root electrotonus in spinal motoneurons. J. gen. Physiol., 35, 289-321 (1951).

10) McIntyre, A.K., Bradley, K., and Brock, L.G.: Responses of motoneurons undergoing chromatolysis. J. gen. Physiol., 42, 931-958 (1959).

11) Nelson, P.G., and Burke, R.E.: Delayed depolarization in cat spinal motoneurons. Experimental Neurology, 17, 16-26 (1967).

12) Terzuolo, C.A., and Araki, T.: Analrsis of intra- vs. extracellular potential changes associated with activity of single spinal motoneurons. Ann. N.Y. Acad. Sci., 94, 547-558 (1961).

13) Tokizane, T.: Studies on the paradoxical phase of sleep in the cat. Progress in Brain Research. 21B (Eds. by T. Tokizane and J.P. Schade), 230-268 (1966). Elsevier Pub. Co., Amsterdam. 